\title{
CHEMISTRY
}

DOI https://doi.org/10.30525/978-9934-26-006-3-33

\section{SOLAR CELLS WITH SILICON NANOWIRES AND METAL NANOPARTICLES ON SURFACE}

\author{
Havryliuk O. O. \\ Candidate of Physical and Mathematical Sciences, \\ Senior Researcher \\ Chuiko Institute of Surface Chemistry \\ of the National Academy of Sciences of Ukraine \\ Kyiv, Ukraine
}

To achieve a greater efficiency of converting light energy into electricity the texture of the surface of the solar cell is used. Anisotropic etching of silicon substrates, which forms a silicon nanowires, is a key process for creating highly efficient and cheap solar cells..

The task of efficient light trapping is very important especially in case of thinner Si substrates. For this in addition to anisotropic texturing of the wafer surface in conventional SCs the other approaches are under intensive investigation. Fine surface structures, comprising features on the nanometer scale can provide excellent light trapping (antireflective) performance [1, p. 6842].

Silicon nanowires (SiNWs) array were fabricated on of p-type (100), one-sided polished silicon substrates and with specific resistance of 10 $\mathrm{Ohm} \times \mathrm{cm}$. Before etching process the samples were sequentially purified in isopropyl alcohol (IPA), deionized water (DW), peroxide-sulfur solution $\left(\mathrm{H}_{2} \mathrm{SO}_{4}: \mathrm{H}_{2} \mathrm{O}_{2}=1: 1\right.$ (vol.), $\left.10 \mathrm{~min}\right)$ with followed washing in DW. After that, for the removal of natural oxides, the samples were immersed in $40 \%$ $\mathrm{HF}$ solution for $2 \mathrm{~min}$. The $\mathrm{Si}$ nanowires were formed by metal-assisted chemical etching (MecEtch) of silicon in $\mathrm{HF} / \mathrm{H}_{2} \mathrm{O}_{2}$ solution [3, p. 145].

In our experiments the silver particles were deposited in the solution of $0.02 \mathrm{M} \mathrm{AgNO} \mathrm{An}_{3} / 4.6 \mathrm{M} \mathrm{HF}$ for $2 \mathrm{~min}$, the silicon wafer was etched in the solution of $0.15 \mathrm{M} \mathrm{H}_{2} \mathrm{O}_{2}$ 
The SEM images of obtained Si NWs are presented in Fig. 1 . The diameters of the nanowires are in the range $60-160 \mathrm{~nm}$ and lengths are 2100-2300 nm.


Fig. 1. SEM images of obtained Si NWs:

(a) top view, (b) cross section view

For numerical calculations of the obtained structures, we used FiniteDifference Time-Domain method (FDTD). The FDTD method is a powerful numerical algorithm for direct solution of Maxwell's equations [2, p. 144].

As the diameter of the nanowires increases, the transmittance in the shortwave region decreases. There is also a clear decrease in the transmittance at the plasmon resonance frequency. For the nanowire diameter of $100 \mathrm{~nm}$, the peak of the plasmon resonance is at a wavelength of $860 \mathrm{~nm}$. As the nanowires diameter increases, this peak shifts from $840-850 \mathrm{~nm}$ (for nanowires with a diameter of 50-80 nm) to $860 \mathrm{~nm}$ (for nanowires with a diameter of $100 \mathrm{~nm}$ ) (fig. $2 \mathrm{a}, \mathrm{b}$ ).

The resonant frequency of metal nanoparticles depends on the size, shape, material of the particles, and the refractive index of the environment. In practice, larger-sized particles applied to the substrate typically lose spherical properties and, therefore, resemble more ellipsoids, which causes the resonance offset frequency to change due to shape changes.

It should be noted that for a structure with $100 \mathrm{~nm}$ nanowires and a diameter of $50 \mathrm{~nm}$ nanoparticles at the plasmon resonance frequency, the 
transmittance is at least $\sim 20 \%$ when for other combinations of the structure it is $\sim 40-50 \%$ (fig. 2).
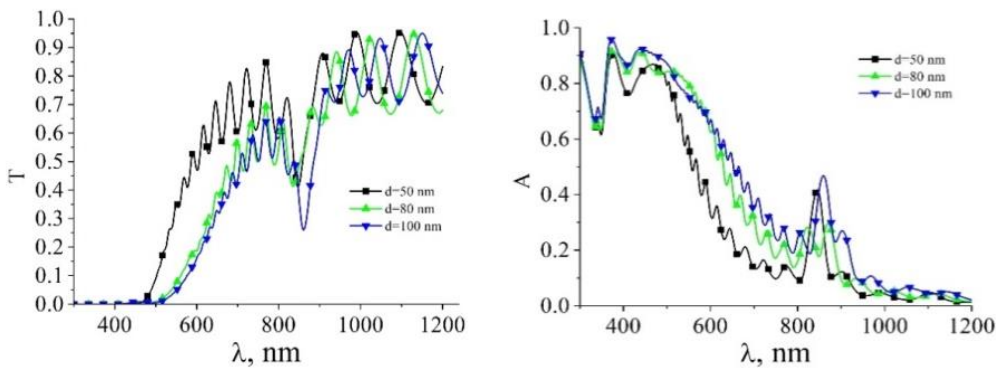

Fig. 2. Transmission (a) and absorption (b) spectra of the structure with silver nanoparticles on the surface of silicon nanowires. (d - diameter of nanowires, diameter of nanoparticles is $50 \mathrm{~nm}$, length of nanowires $2300 \mathrm{~nm}$ )

The theoretical modeling and experimental investigations of Si NWs SC allowed to determine their optical properties. The simulation of reflection, absorption and transmission of light in Si-NWs arrays in dependence on their parameters, namely: diameter, length and period allowed to demonstrate the main optical properties of Si-NWs. There was strong influence of nanowire diameter and period on reflectance of light. But the effect of length wasn't remarkable in investigated range. It has been found that, in comparison to Si films, the nanowire array has decreased reflection in all spectral range. In region of long wavelength the extinction coefficient of silicon is small and interference effects exist, resulting in oscillation of reflectance and transmittance.

\section{References:}

1. Hung Y.J., Lee S.L., Coldren L.A. Deep and tapered silicon photonic crystals for achieving antireflection and enhanced absorption. Opt Express. 2010. № 18(7). P. 6841-6852.

2. Pylypova O.V., Evtukh A.A., Parfenyuk P.V., Ivanov I.I., Korobchuk I.M., Havryliuk O.O., Semchuk O.Yu. Electrical and Optical Properties of Nanowires Based Solar Cell with Radial p-n Junction. Opto-Electronic Review. 2019. № 27(2). P. 143-148. 